\title{
Internationale Aktivitäten im Fachgebiet Logistik
}

\author{
Bernd Hentschel
}

\section{Einleitung}

Mit dem Aufbau des Studienganges „Logistik“ an der Technischen Fachhochschule Wildau und seiner Ausrichtung auf „Unternehmenslogistik“ sowie „Internationale Logistik" wurden seit 1996 Logistikkonferenzen zwischen polnischen und deutschen Logistikpartnern unter Führung der Technischen Fachhochschule Wildau organisiert und durchgeführt. Ziel dieser Logistikkonferenzen war es, den Wissenstransfer zu Logistikproblemen in Richtung Osteuropa zu organisieren sowie gemeinsame Projekte zwischen deutschen und polnischen Hoch- und Fachschulen sowie Unternehmen zu initiieren. Dazu wurde die 1. Deutsch-Polnische Logistikkonferenz im Jahre 1996 in Wildau unter dem Thema „Entwicklungen logistischer Dienstleistungen und ihre Nutzung in den neuen Bundesländern und Polen" durchgeführt. Es zeigte sich, dass eine große Resonanz zu verzeichnen war. Deshalb wurde gemeinsam mit den mitveranstaltenden Partnern aus Polen - Institut für Logistik und Lagerwesen Poznan sowie Technische Universität Poznan - beschlossen, alternierend in jedem Jahr eine weitere Konferenz im jeweiligen Partnerland zu organisieren. 1997 wurde dann die 2. PolnischDeutsche Logistikkonferenz zum Themenkomplex „Logistikinnovationen für Produkte und Dienstleistungen in der Wirtschaft" in Poznan erfolgreich absolviert. Die 3. Deutsch-Polnische Konferenz fand 1998 in Wildau zum Komplex „Informations- und Kommunikationstechniken in Logistikprozessen" statt. Aufgrund des erreichten Standes wurde gemeinsam mit dem Land Brandenburg entschieden, diese Konferenzen als untrennbaren Bestandteil in die jährlich durchzuführenden Brandenburgischen Wirtschaftswochen zu integrieren.

Es wurde ab 1999 die 4. Polnisch-Deutsche Logistikkonferenz zum Komplex „Innovative Grenzlogistik“ zweigeteilt im Grenzort Slubice sowie in Poznan organisiert und mit gleichfalls großem Erfolg abgeschlossen. Aus letzterer Konferenz zur innovativen Grenzlogistik entstand auf Empfehlung des Ministerpräsidenten Dr. Stolpe ein gemeinsames grenzüberschreitendes Logistikprojekt zu einem „Virtuellen Deutsch-Polnischen Logistik-Broker-System". Gemeinsam mit den polnischen Partnern wurde dieser umfassende Komplex inhaltlich strukturiert und das Konzept zur 5. Deutsch-Polnischen Logistikkonferenz einem breiten Teilnehmerkreis vorgestellt.

Auf Basis der weiteren Aktivitäten zur Internationalisierung der Logistikausbildung an der Technischen Fachhochschule Wildau wurden ab 1999 die Kontakte bis nach Brasilien in fachlicher und ausbildungsseitiger Hinsicht erweitert. In Übereinstimmung mit den Orga- nisatoren der Brandenburgischen Wirtschaftswoche wurde dann entschieden, am 28. September 2000 die 1. Internationale Logistikkonferenz mit Beteiligung europäischer und südamerikanischer Partner durchzuführen. Das Thema dieser Internationalen Logistikkonferenz passte sich die thematischen Zielstellung an und lautete "Logistikkooperationen im globalen Markt". Mit 220 anwesenden Teilnehmern wurde die Konferenz zu einem überragenden Erfolg für die Technische Fachhochschule Wildau und schuf neue internationale Verbindungen bzw. führte zur weiteren Intensivierung internationaler Logistikprojekte.

\section{Internationale Logistikkonferenz an der Technischen Fachhochschule Wildau}

Diese Konferenz stand unter der Schirmherrschaft des Ministerpräsidenten des Landes Brandenburg, Dr. Manfred Stolpe. Die Internationalität im Rahmen der Plenarveranstaltung wurde dadurch dokumentiert, dass über Videokonferenz länderübergreifende Videokonferenzschaltungen aufgebaut wurden und somit die modernste Kommunikationstechnik bei der Konferenz zur Anwendung gelangte. In der nachfolgenden Abbildung verdeutlicht ein Ausschnitt die gemeinsame Begrüßung aller Konferenzteilnehmer durch Ministerpräsident Stolpe sowie den Marschall von Wielkopolska, Herrn Mikolajczak.

Seitens des Landes Brandenburg wurde vom damaligen Minister für Wissenschaft, Forschung und Kultur, Dr. Wolfgang Hackel, die Begrüßungsrede gehalten und die strategische Ausrichtung der Technischen Fachhochschule Wildau als Kompetenzzentrum für Logistik zu unseren

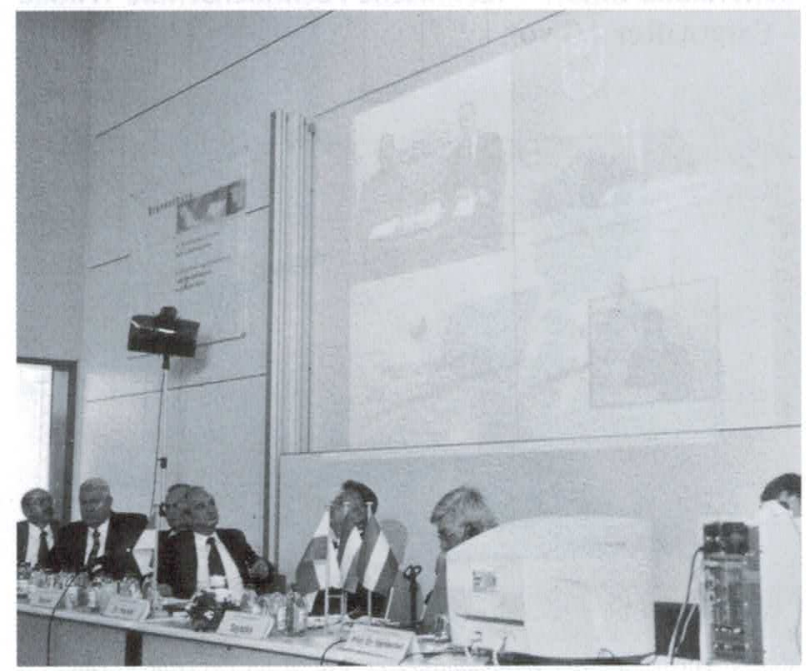

Abb. 1: Begrüßung der Teilnehmer über Videokonferenz 
osteuropäischen Partnern erläutert. Minister Hackel würdigte die bisherigen Aktivitäten im nationalen wie auch internationalen Rahmen und sagte die Unterstuitzung des Landes Brandenburg zu. Insbesondere regte er an, die Logistikkooperationen mit den Partnern aus den Niederlanden und Brasilien sowie die bestehende Kooperation mit Polen weiter intensiv zu gestalten, zumal hier bereits außerordentlich gute Ausbildungsmöglichkeiten im gegenseitigen Studentenaustausch gegeben waren.

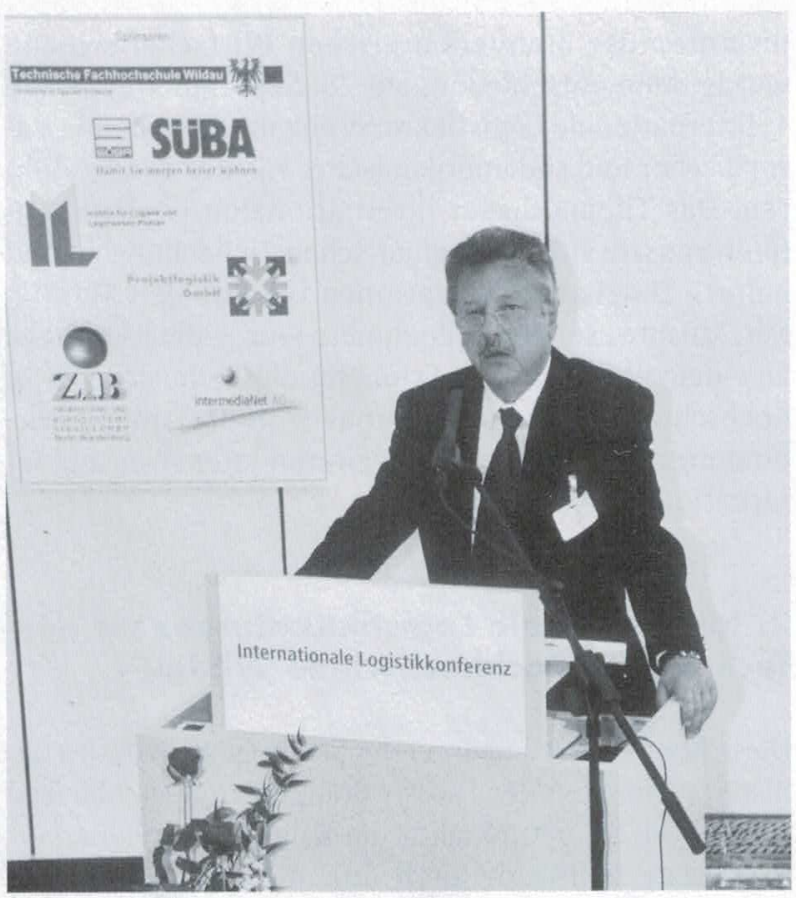

Abb. 2: Begrüßungsrede Dr. Wolfgang Hackel

Renommierte Industrievertreter waren zu dieser Konferenz erschienen und konnten über bestehende Logistikkooperationen mit der Technischen Fachhochschule Wildau berichten. Dazu gehörte das größte deutsche Wohnungsbauunternehmen SÜBA AG mit der SGB SÜBA Generalbau GmbH und seinem Geschäftsfuihrer, Prof. Dr. Heinz Zerressen, an der Spitze. Dieses Unternehmen hatte sich in der Vergangenheit besonders durch konkrete Aufträge für den Logistikfachbereich eingebracht. Zerressen stellte aus Sicht des Bauwesens das neueste Kooperationsprojekt zwischen dem Verbund SGB SÜBA Generalbau GmbH - Technische Fachhochschule Wildau - CargoLifter AG vor.

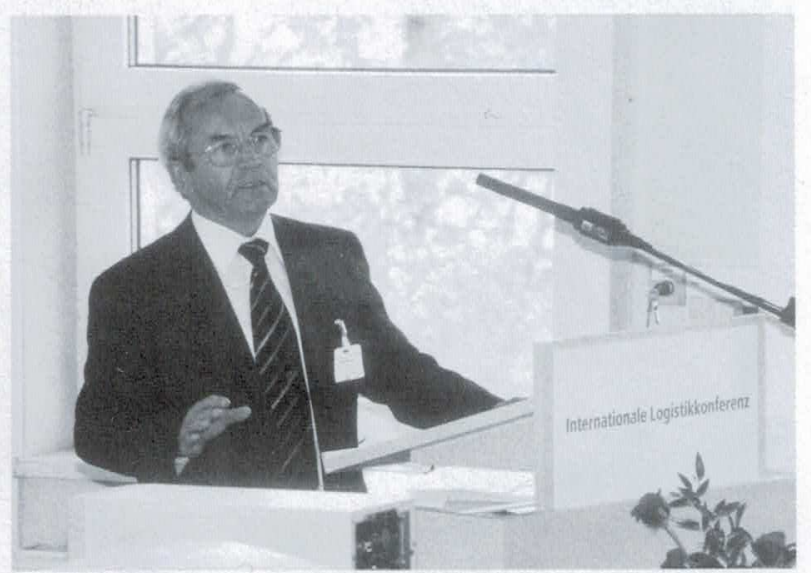

Abb. 3: Vorstellung des Verbundprojektes SGB SÜBA Generalbau GmbH Technische Fachhochschule Wildau - CargoLifter AG durch Prof. Dr. Zerressen
Vor der Technischen Fachhochschule Wildau steht ihrerseits die Herausforderung, eine überflurgefuihrte Materialflusslogistiklösung unter Nutzung des CargoLifters zum Transport von Hauselementen oder -segmenten deutschlandweit zu planen und zu realisieren. Dieses Projekt weckte auch das Interesse der brasilianischen Partner, wie der Rektor der Ingenieurhochschule Belo Horizonte, Prof. Dr. Paulo Henrique, in seinem Vortrag bekundete. Aus diesem Vortrag ergab sich im Nachhinein eine entsprechende Kooperation zwischen der CargoLifter AG und Partnern in Brasilien, die auf die Initiative der Technischen Fachhochschule Wildau und diese Logistikkonferenz zurüickzuführen waren. In Abbildung 4 trägt der Rektor der Ingenieurhochschule Belo Horizonte, Professor Henrique, das Konzept logistischer Aktivitäten für Brasilien vor.

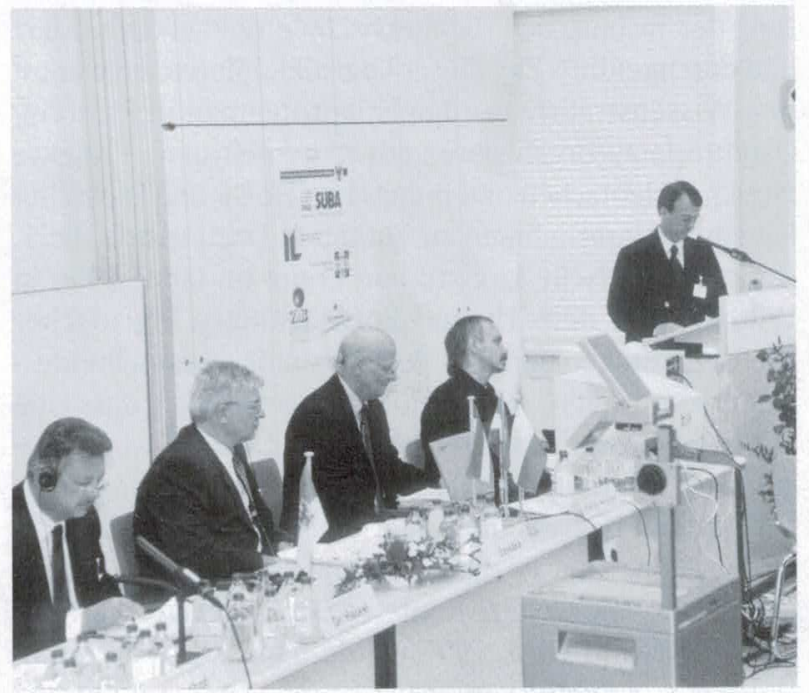

Abb. 4: Professor Paulo Henrique von der Ingenieurhochschule Belo Horizonte beim Vortrag

Als weiteres überragendes Logistikprojekt im grenzüberschreitenden Verkehr wurde durch den Hauptprojektleiter, Dipl.-Ing. Hans-Joachim Bischof das „Virtuelle Deutsch-Polnische Logistik-Broker-System“ vorgestellt. Dieses große Verbundvorhaben im Wertumfang mehrerer Millionen D-Mark wird unter Führung der Technischen Fachhochschule Wildau sowie des polnischen Institutes für Logistik und Lagerwesen Pozna 'n, den Firmen Projektlogistik GmbH, PSI Transportation $\mathrm{GmbH}$ sowie EPV Europrojekt Verkehr GmbH in den nächsten zwei Jahren erarbeitet. Dieses Projekt wird im Status eines EG-Projektes bearbeitet und soll unter Einsatz modernster Informations- und Kommunikationstechniken die logistischen Abläufe internationaler Verkehrsdienste sowie Logistikketten zur Straße und zur Schiene grenzuiberschreitend optimieren. Dazu wird am Logistikstandort POINT 36 Enterprises eine entsprechende Broker-Lösung installiert, die alle ankommenden Transporte aus den westeuropäischen Bereichen in den osteuropäischen Bereich sowie umgekehrt nach optimalen Kriterien steuern soll. Dieses Projekt wurde in einer zirka halbjährigen Vorbereitungsphase von den Partnern strukturiert und mit dieser Logistikkonferenz initiiert.

Abbildung 5 zeigt den polnischen Vertreter, mgr. inz. Grzegorz Szyszka, bei seinen Ausführungen. 


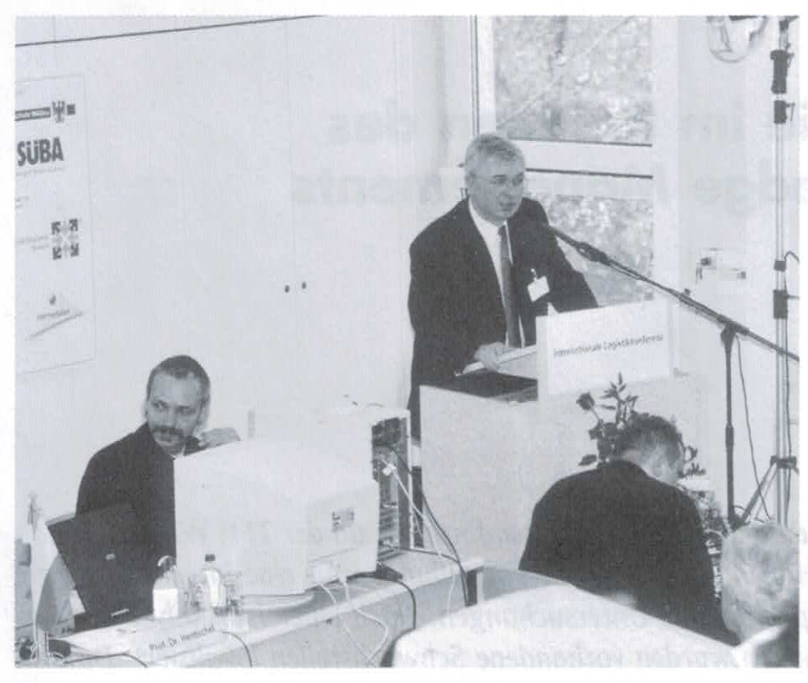

Abb. 5: Rede von mgr. inz. Grzegorz Szyszka aus Poznań

Traditionell gehört zur Logistikkonferenz auch die jährliche Verleihung des studentischen Logistikpreises der Technischen Fachhochschule Wildau. Diese Preisverleihung wurde durch den Geschäftsführer der SGB SÜBA Generalbau GmbH, Prof. Dr. Heinz Zerressen, vorgenommen. Es wurden studentische Teams ausgezeichnet, die eine ausgezeichnete logistische und betriebswirtschaftliche Konzeptvariante für den Einsatz des CargoLifters im überflurgeführten Transport für das Bauwesen erarbeitet. Der Preis wurde durch das auszeichnende Unternehmen finanziell gesponsert, so dass ein wirksamer Anreiz für die Erarbeitung innovativer Logistikkonzeptionen gegeben war.

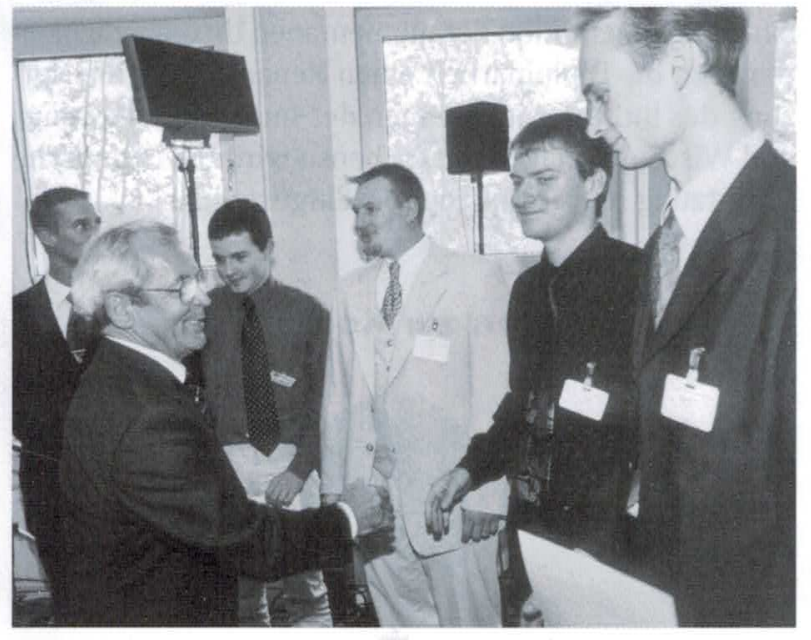

Bild 6: Auszeichnung eines studentischen Teams

Um die Internationalität abzurunden, schloss die Konferenz mit einer 60-minütigen Live-Fernsehdiskussion zu dem Generalthema der Konferenz im FAB-Fernsehen. In einer sehr interessanten gemischten Runde diskutierten die Vizepräsidentin der TFH Wildau, Prof. Dr. Asta Richter, der Deutsche Honorarkonsul in Belo Horizonte, Hans Kampik, Herr Glahr von der CargoLifter Network GmbH, Prof. Dr. Heinz Zerressen von der SGB SÜBA Generalbau GmbH, Prof. Dr.-Ing. Bernd Hentschel, TFH Wildau, Hans-Joachim Bischof von der EPV Europrojekt Verkehr GmbH und Studenten sowie Zuhörer aus dem Auditorium. Es wurde zum Abschluss durch alle Beteiligten eingeschätzt, dass der von der
Technischen Fachhochschule Wildau eingeschlagene generelle Weg der internationalen Logistikausbildung kontinuierlich fortgeführt werden sollte sowie der Ausbau internationaler Logistikbeziehungen aktiviert werden muss. Das Potenzial unserer Studenten bietet ein ausgezeichnetes Fundament, diesen Gedanken zu realisieren. Alle Teilnehmer waren sich einig, dass zu einer weiteren internationalen Konferenz, dann in der Republik Polen, bereits über Ergebnisse aus dieser neuen Qualität gesprochen werden kann.

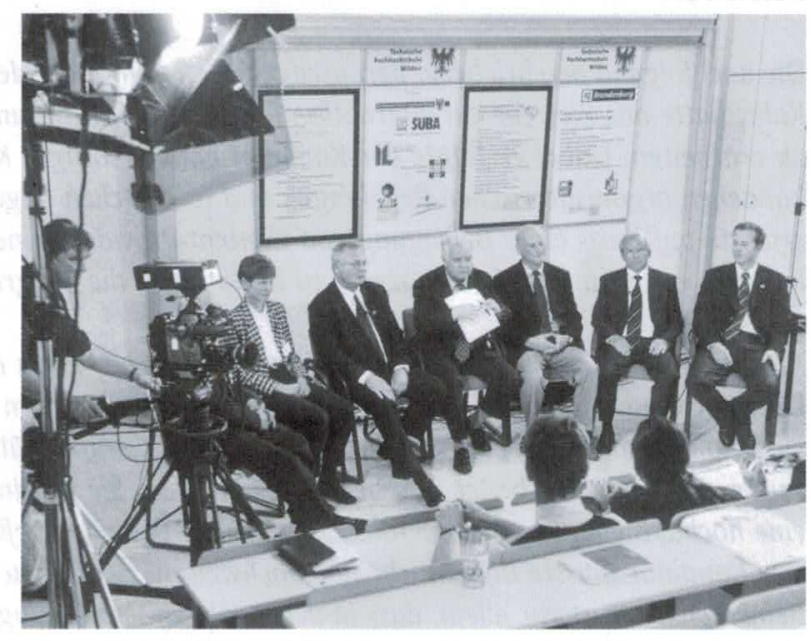

Abb. 7: Fernsehdiskussionsrunde mit den Teilnehmern (v.l.n.r.) Hans Joachim Bischof, EPV (verdeckt), Prof. Dr. Asta Richter, TFH Wildau, Hans Kampik, Honorarkonsul Belo Horizonte, Herr Dr. Büchel, Moderator, Prof. Dr. Bernd Hentschel, TFH Wildau, Prof. Dr. Heinz Zerressen, SGB SÜBA Generalbau $\mathrm{GmbH}$, Herr Glahr, CargoLifter Network GmbH

\section{Zusammenfassung}

Mit dem 10-jährigen Jubiläum der Technischen Fachhochschule Wildau gestaltet sich auch die Entwicklung im Fachgebiet Logistik positiv, und mit fünf durchgeführten internationalen Logistikveranstaltungen hat sich bereits eine Tradition in den internationalen Logistikbeziehungen herauskristallisiert. Dieser Weg sollte sofern die nicht unerheblichen Aufwändungen fuir derartige Konferenzen durch die Technische Fachhochschule Wildau aufgebracht und eine konstruktive Unterstiitzung gesichert werden können - konsequent weiterverfolgt werden. Nur so lässt sich im globalen Wettbewerb die Region Wildau mit der Technischen Fachhochschule und ihrem Logistikpotenzial auch logistisch richtig in Szene setzen.

\section{Autor}

Prof. Dr.-Ing. habil. Bernd Hentschel

Technische Fachhochschule Wildau Fachbereich Ingenieur-/Wirtschaftsingenieurwesen Institut für Unternehmenslogistik Tel. (0 33 75) 508-927

E-Mail: bhentsch@wi-bw.tfh-wildau.de 